\title{
Propiedades psicométricas de la Escala de Estilos Parentales (MOPS)
}

\author{
María Matalinares \\ Ornella Raymundo \\ Deyvi Baca \\ Universidad Nacional Mayor de San Marcos
}

Recibido: 15 de mayo de 2014 / Aprobado: 15 de junio de 2014

Se analizó la validez y confiabilidad de la Escala de Estilos Parentales

Disfuncionales (MOPS, por sus siglas en inglés), de Parker, Roussos, HadziPavlovic, Mitchell, Wilhelm y Austin (1997), con baremos peruanos. La muestra estuvo conformada por 2370 adolescentes de 13 a 19 años (47,6\% de género masculino, 52,4\% de género femenino), 56,5\% en edades entre 15 y 16 años, que cursan el tercero (32\%), cuarto (34,2\%) y quinto (33,8\%) de secundaria de 14 ciudades representativas de la costa, sierra y selva del Perú. La escala mostró una confiabilidad moderada de.87, en la subescala de estilos parentales del padre, así como se extrajo la confiabilidad de .80 en la subescala de estilos parentales de la madre. En el análisis factorial exploratorio se obtuvieron 3 factores; indiferencia, abuso y sobreprotección.

estilos parentales / disfuncionalidad / adolescentes / crianza

\section{Psychometric properties of the Measure of Parental Style (MOPS)}

This objective of this study was to analyze the validity and reliability of the Measure of Parental Style (MOPS) scale created by Parker, Roussos, HadziPavlovic, Mitchell, Wilhelm y Austin (1997) with Peruvian scales. The sample consisted of 2370 adolescents between 13 and 19 years (47,6 males, 52,4 females), $56,5 \%$ between 15 and 16 years, $32 \%$ were enrolled in third year secondary schooling, $34 \%$ in fourth, and 33,8\% in fifth. The sample was drawn from 14 cities of the different regions in Peru. Results showed that the instrument has moderate reliability of .87 in the subscale Father Parenting Styles, and reliability of .80 in the subscale Mother Parenting Styles. The Exploratory Factor Analysis identified 3 factors: indifference, abuse and overprotection. 
El ser humano, al nacer, es aún inmaduro y requiere de constante asistencia por parte de sus cuidadores; además, presenta expectativas respecto a las respuestas provenientes de sus figuras de apego frente a sus necesidades básicas y afectivas. Estas interacciones conforman el desarrollo de representaciones cognitivas denominadas "modelos internos de funcionamiento" (Bowlby, 1969). Dichos modelos consisten en creencias organizadas que forman expectativas, percepciones, reacciones y conductas esenciales en la configuración de la forma en que opera el sistema de apego durante toda la vida, lo que constituye la base del estilo de apego de un individuo (Collins \& Read, 1990; Fraley \& Shaver, 2000; Mikulincer \& Shaver, 2007; citados en Bedoya, 2012).

El estilo parental es una constelación de actitudes acerca del niño, que le son comunicadas y que, en conjunto, crean un clima emocional en el que se ponen de manifiesto los comportamientos de los padres. Estos comportamientos incluyen tanto las conductas a través de las cuales los padres desarrollan sus propios deberes de paternidad (prácticas parentales) como cualquier otro tipo de comportamientos entre los que se encuentran los gestos, cambios en el tono de voz, expresiones espontáneas de afecto, etc. (Darling \& Steinberg, 1993; citado en Estévez, Murgui, Musiti \& Moreno, 2008).

La familia, por una parte, representa el eje central del ciclo vital; se trata de una institución social fundamentada en relaciones afectivas, desde la cual el niño y el adolescente aprehenden los valores, creencias, normas y formas de conducta apropiadas para la sociedad a la que pertenecen. Este proceso tiene lugar en un ambiente o clima social que se define como el ambiente percibido e interpretado por los miembros que integran una organización y que, a su vez, ejerce una importante influencia en el comportamiento de los integrantes de ese contexto, así como en su desarrollo social, físico, afectivo e intelectual (Sánchez-Queija, 2007).

Según las predicciones de Bowlby (1973), eventos vitales estresantes y traumáticos durante la infancia (pérdidas parentales, separación, dificultades de salud física o mental, maltrato, etc.) conducirían a importantes cambios en las representaciones de apego. Existen numerosas investigaciones que señalan la estrecha relación existente entre la percepción de un clima positivo en estos contextos y el ajuste personal en la adolescencia, entendido este como un ajuste integral del individuo en los ámbitos emocional y conductual (Oliva, Parra \& Sánchez, 2002; citado en Fourment, 2009).

En tal sentido, los estilos parentales son importantes, ya que el ser humano aprende las formas de vincularse a las personas que, a futuro, regirán en su interacción social. Sin embargo, así como se puede brindar vínculos de apego saludables, de cuidado y protección, también se pueden generar estilos pa- 
rentales disfuncionales que en el futuro producirán problemas en la interacción social del individuo; es más, se ha demostrado que los estilos parentales son predictores de enfermedades psiquiátricas. En efecto, la teoría de Bowlby (1977) explica que la educación anómala se manifiesta cuando los padres fallan en proporcionar el cuidado, por ser insensibles, manifestar menosprecio, rechazo, o bien por tener una sobreprotección o un control excesivos.

Estas actitudes y comportamientos paternales son considerados como predisponentes en un niño al desorden psiquiátrico, así como a interacciones disfuncionales sociales y/o emocionales en la edad adulta (Parker et al., 1997).

Parker, Tupling y Brown (1979) consideran que hay una significativa asociación entre parentalidad disfuncional (padres poco afectuosos y sobreprotectores) y los trastornos psiquiátricos. Estos autores identificaron tres tipos de vínculos parentales disfuncionales: indiferencia, abuso y sobreprotección. En el primer estilo, la indiferencia se caracteriza por la ausencia de exigencias y de cumplimiento de responsabilidades, falta de estructuración, control y apoyo. Estos padres, generalmente, desvían sus responsabilidades paternas hacia otras figuras como el colegio u otros familiares; sus hijos presentan problemas de conducta, el ambiente familiar suele ser desorganizado y son altamente vulnerables a la ruptura familiar (Steinber, Blatt-Eisengart \& Cauffman, 2006;
Raya, 2008). El abuso -el segundo vínculo parental disfuncional- es un patrón estricto de crianza en el que los padres imponen las reglas para sus hijos, esperan una obediencia estricta y se basan en el poder que tienen como padres (Baumrind, 2005); por lo general, se generan castigos físicos y verbales. Finalmente, la sobreprotección se caracteriza por combinar bajo control y exigencia con poca sensibilidad hacia las necesidades de los hijos; muchas veces pueden ser crueles y no establecen normas, no se muestran firmes frente a sus hijos, no esperan acciones maduras de sus hijos. La comunicación no es efectiva y suele ir en una sola dirección: del padre al hijo (Baumrind, 2005).

Paker, Tupling y Brown, en 1979, crearon un instrumento para medir el apego basado en la teoría de Bowlby (Parental Bonding Instrument, PBI), el cual propone dos dimensiones: el cuidado y la protección. Este test evalúa la percepción que tienen los adultos de los cuidados brindados por sus padres en sus dieciséis primeros años. Así como esta escala, se puede apreciar en la tabla 1 los diferentes instrumentos que miden el apego creados a lo largo del tiempo.

El presente estudio se realizó con el objeto de proporcionar a los psicólogos peruanos una versión traducida, válida y confiable de la Escala de Estilos Parentales (MOPS, por sus siglas en inglés), propuesta por Paker, Roussos, Hadzi-Pavlovic, Mitchell, Wilhelm y Austin (1997). 
Tabla 1

Principales instrumentos de evaluación del apego en adultos

\begin{tabular}{|c|c|c|c|}
\hline Escala & $\begin{array}{l}\text { Número de } \\
\text { ítems }\end{array}$ & Tipo de relación & Referencia \\
\hline Adult Attachment Styles & 1 & Relaciones íntimas & Hazan y Shaver (1987) \\
\hline $\begin{array}{l}\text { Adult Attachment Questionnaire } \\
\text { (AAQ) }\end{array}$ & $\begin{array}{l}17 \\
13\end{array}$ & Pareja & $\begin{array}{l}\text { Simpson (1990) } \\
\text { Simpson, Rholes y Phillips } \\
\text { (1996) }\end{array}$ \\
\hline $\begin{array}{l}\text { Avoidant Attachment Questionnaire } \\
\text { for Adults (AAQA) }\end{array}$ & 22 & General & $\begin{array}{l}\text { West y Shlertdon-kellor } \\
(1992)\end{array}$ \\
\hline $\begin{array}{l}\text { Adult Attachment Scale (AAS) y } \\
\text { Revised Adult Attachment Scale } \\
\text { (RAAS) }\end{array}$ & $\begin{array}{l}21 \\
18\end{array}$ & Pareja & $\begin{array}{l}\text { Collins y Read (1990) } \\
\text { Collins (1996) }\end{array}$ \\
\hline $\begin{array}{l}\text { Attachment History Questionnaire } \\
\text { (AHQ) }\end{array}$ & 51 & Pareja & Pottharst (1990) \\
\hline $\begin{array}{l}\text { Attachment and Object Relations } \\
\text { Inventory (AORI) }\end{array}$ & 75 & $\begin{array}{l}\text { Padres, compa- } \\
\text { ñeros, parejas y sí } \\
\text { mismo }\end{array}$ & $\begin{array}{l}\text { Buelow, McClain y Mclntosh } \\
\text { (1996) }\end{array}$ \\
\hline $\begin{array}{l}\text { Attachment Style Questionnaire } \\
\text { (ASQ) }\end{array}$ & 40 & $\begin{array}{l}\text { Relaciones cer- } \\
\text { canas }\end{array}$ & $\begin{array}{l}\text { Feeney, Noller y Hanrahan } \\
\text { (1994) }\end{array}$ \\
\hline Continued Attachment Scale (CAS) & 12 & Padres & $\begin{array}{l}\text { Berman, Heiss y Sperling } \\
\text { (1994) }\end{array}$ \\
\hline $\begin{array}{l}\text { Client Attachment to Therapist Scale } \\
\text { (CATS) }\end{array}$ & 36 & Terapeuta & $\begin{array}{l}\text { Mallinckrodt, Gantt y Coble } \\
\text { (1995) }\end{array}$ \\
\hline $\begin{array}{l}\text { Experiences in Close Relationships } \\
\text { (ECR) y Experiences in Close } \\
\text { Relationships Revised (ECR-R) }\end{array}$ & 36 & Pareja (o general) & $\begin{array}{l}\text { Brennan, Clark y Shaver } \\
\text { (1998) } \\
\text { Fraley y Shaver (2000) }\end{array}$ \\
\hline $\begin{array}{l}\text { Measure of Attachment Qualities } \\
\text { (MAQ) }\end{array}$ & 14 & General & Carver (1997) \\
\hline Mother-Father-Peer Scale (MFPS) & 70 & $\begin{array}{l}\text { Padres y } \\
\text { compañeros }\end{array}$ & Epstein (1983) \\
\hline $\begin{array}{l}\text { Maternal Separation Anxiety Scale } \\
\text { (MSAS) }\end{array}$ & 35 & Niño & $\begin{array}{l}\text { Hock, McBride y Gnezda } \\
\text { (1989) }\end{array}$ \\
\hline $\begin{array}{l}\text { Parental Attachment Questionnaire } \\
\text { (PAQ) }\end{array}$ & 55 & $\begin{array}{l}\text { Padres (de } \\
\text { adolescentes) }\end{array}$ & Kenny (1987) \\
\hline $\begin{array}{l}\text { Parents of Adolescents Separation } \\
\text { Anxiety Scale (PASAS) }\end{array}$ & 35 & $\begin{array}{l}\text { Adolescentes y } \\
\text { niños }\end{array}$ & $\begin{array}{l}\text { Hock, Eberly, Bartle-Haring, } \\
\text { Ellwanger y Widaman (2001) }\end{array}$ \\
\hline
\end{tabular}




\begin{tabular}{|c|c|c|c|}
\hline & & & $\begin{array}{l}\text { Parker, Tupling y Brown } \\
\text { (1979) }\end{array}$ \\
\hline Parenting Bonding Instrument (PBI) & 50 & Padres & $\begin{array}{l}\text { Paker, Roussos, Hadzi- } \\
\text { Pavlovic, Mitchell, Wilhelm, y } \\
\text { Austin (1997) }\end{array}$ \\
\hline $\begin{array}{l}\text { Reciprocal Attachment } \\
\text { Questionnaire for Adults (RAQA) }\end{array}$ & 15 & $\begin{array}{l}\text { Figura de apego } \\
\text { más importante }\end{array}$ & $\begin{array}{l}\text { West, Sheldon y Reiffer } \\
\text { (1987) } \\
\text { West y Sheldon (1988) }\end{array}$ \\
\hline Relationship Questionnaire (RQ) & 4 & Pareja & $\begin{array}{l}\text { Bartholomew y Horowitz } \\
\text { (1991) }\end{array}$ \\
\hline $\begin{array}{l}\text { Relationship Scales Questionnaire } \\
\text { (RSQ) }\end{array}$ & 30 & Pareja & $\begin{array}{l}\text { Griffin } \\
(1994)\end{array} \quad$ B Bartholomew \\
\hline $\begin{array}{l}\text { Revised Inventory of Parental } \\
\text { Attachment (R-IPA) }\end{array}$ & 30 & Niños & $\begin{array}{l}\text { Johnson, Ketring y Abshire } \\
\text { (2003) }\end{array}$ \\
\hline $\begin{array}{l}\text { Vulnerable Attachment Style } \\
\text { Questionnaire (VASQ) }\end{array}$ & 23 & Apoyo & $\begin{array}{l}\text { Bifulco, Mahon, Kwon, } \\
\text { Moran y Jacobs (2003) }\end{array}$ \\
\hline
\end{tabular}

Fuente: Bedoya (2012).

La importancia de este trabajo radica en que se puede medir el apego, con una orientación hacia la identificación de estilos parentales disfuncionales, tales como la indiferencia, el abuso y la sobreprotección, y asociar dichas dimensiones a trastornos mentales. De esta manera, el MOPS puede ser utilizado de forma independiente del Parental Bonding Instrument (PBI), lo que ofrece más alternativas para evaluar los estilos parentales percibidos.

\section{MÉtodos}

En la línea de Sánchez y Reyes (2009), el presente estudio es de tipo tecnológico, ya que pretende aportar un instrumento válido para posteriores investigaciones.

\section{Participantes}

La muestra estuvo conformada por 2370 adolescentes de 13 a 19 años. La mayor parte de ellos (el 56,5\%) se encuentra en edades entre 15 y 16 años, y cursan el tercero $(32 \%)$, cuarto $(34,2 \%$ ) y quinto $(33,8 \%)$ de secundaria de 14 ciudades representativas de la costa, sierra y selva del Perú: Tumbes, Trujillo, Lima, Tacna, Huánuco, Huancayo, Huanta, Huancavelica, Cusco, Cerro de Pasco, Huaraz, Bagua Grande, Moyobamba y Pucallpa. Asimismo, el 47,6 \% fueron de género masculino y el $52,4 \%$ de género femenino (ver anexo 1).

\section{Intrumento}

Es el instrumento diseñado por Parker et al. (1997) para identificar los esti- 
los de crianza disfuncionales en los dieciséis primeros años de la vida de un adolescente y/o adulto. Los participantes evalúan cada ítem como una descripción, por separado, del comportamiento de su madre y de su padre hacia ellos en sus dieciséis primeros años. Esta escala deviene del Parental Bonding Instrument (PBI), que mide el cuidado y la protección; los autores crearon el MOPS (Measure of Dysfunctional Parenting Style) con la finalidad de refinar la medida de los estilos parentales. Este instrumento incluye tres escalas: indiferencia, abuso y sobrecontrol (ver anexo 7). La prueba obtuvo la consistencia interna de .829 en la dimensión de estilos parentales del padre, mientras que la consistencia interna de la dimensión de los estilos parentales de la madre fue de .781, ambas aceptables y confiables, ya que dichos datos fueron obtenidos por los autores originales; con el presente estudio se brinda una estandarización nacional del instrumento. La teoría que sostiene el MOPS está basada en Bowlby (1977, citado por Melis, Dávila, Ormeño, Vera, Greppi \& Gloger, 2001), quien señala que la educación anómala es aquella donde los padres fallan en proporcionar el cuidado, por ser insensibles, menospreciar, rechazar y sobreproteger de forma excesiva. Estas actitudes y comportamientos paternales son considerados como factores de una predisposición en un niño al desorden psiquiátrico, así como a interacciones disfuncionales sociales y/o emocionales en la edad adulta (Parker et al., 1997).

\section{Resultados}

\section{Análisis de fiabilidad}

La Escala de Estilos Parentales (MOPS) es una prueba con alternativas múltiples de tipo Likert; por tanto, se consideró apropiado utilizar un análisis de consistencia interna a través del coeficiente alfa de Cronbach (Kerlinger \& Lee, 2002). La prueba mencionada está compuesta por dos subescalas: estilos parentales del padre y estilos parentales de la madre, por lo que realizó un análisis de fiabilidad por cada escala.

Con relación a los estilos parentales del padre, se obtuvo un alfa de Cronbach que oscila entre .766 y .851 , lo que estaría indicando un nivel entre bajo y moderado de consistencia interna (Murphy \& Davishofer, citado en Hogan, 2004).

En la tabla 2, se observa en la gran mayoría de ítems correlaciones ítemstest por encima de .200, con la excepción del ítem 1. Por tanto, se estaría indicando que la gran mayoría de ellos poseen índices de discriminación aceptables (Ary, Jacobs \& Razavieh, 1990; citado en Aliaga et al., 2006). El ítem 1 posee un índice de discriminación bajo, por lo que se procede a eliminarlo, lo cual incrementa sensiblemente el alfa de Cronbach (ver anexo 1). 
Tabla 2

Correlaciones ítems-test de la subescala de estilos parentales del padre

\begin{tabular}{ccccc}
\hline Í́tems & Subescala total & \multicolumn{3}{c}{ Región natural } \\
\cline { 3 - 5 } & & Costa & Sierra & Selva \\
\hline Ítem 1 & .007 & .032 & -.009 & .007 \\
Ítem 2 & .507 & .435 & .549 & .448 \\
Ítem 4 & .294 & .242 & .330 & .197 \\
Ítem 5 & .563 & .514 & .620 & .469 \\
Ítem 6 & .589 & .597 & .615 & .526 \\
Ítem 7 & .511 & .462 & .583 & .413 \\
Ítem 8 & .415 & .401 & .471 & .255 \\
Ítem 9 & .398 & .371 & .449 & .273 \\
Ítem 10 & .582 & .498 & .624 & .480 \\
Ítem 11 & .635 & .553 & .674 & .583 \\
Ítem 12 & .467 & .406 & .491 & .467 \\
Ítem 13 & .528 & .531 & .532 & .511 \\
Ítem 14 & .528 & .548 & .548 & .444 \\
Ítem 15 & .465 & .471 & .480 & .373 \\
Alfa de Cronbach & .599 & .515 & .632 & .552 \\
\hline
\end{tabular}

Con respecto a la subescala de estilos parentales de la madre se halló un alfa de Cronbach que oscila entre .738 y .804, lo que estaría indicando un nivel entre bajo y moderado de consistencia interna (Murphy \& Davishofer, citado en Hogan, 2004).

Se observa en la tabla 3 similares correlaciones ítems-test que en la subescala de estilos parentales del padre, donde la mayor parte de los ítems poseen correlaciones por encima de .200, con la excepción del ítem 1. Por lo tan- to, de igual modo se estaría indicando que la gran mayoría de los ítems poseen índices de discriminación aceptables. Asimismo, se procede a eliminar el ítem 1 por sus bajos índices de discriminación (ver anexo 1).

Después de la eliminación del ítem 1, se realizó un segundo análisis de la confiabilidad con los 14 ítems restantes. En él se encontró un alfa de Cronbach para la subescala de estilos parentales del padre de .853 , mientras que para la subescala de estilos parentales de la 
Tabla 3

Correlaciones ítems-test de la subescala de estilos parentales de la madre

\begin{tabular}{ccccc}
\hline \multirow{2}{*}{ Ítems } & Subescala total & \multicolumn{3}{c}{ Región natural } \\
\cline { 3 - 5 } & & Costa & Sierra & Selva \\
\hline Ítem 1 & .063 & .021 & .069 & .090 \\
Ítem 2 & .448 & .409 & .471 & .404 \\
Ítem 3 & .277 & .262 & .289 & .216 \\
Ítem 4 & .467 & .460 & .492 & .452 \\
Ítem 6 & .511 & .472 & .523 & .511 \\
Ítem 7 & .495 & .478 & .538 & .408 \\
Ítem 8 & .332 & .326 & .371 & .264 \\
Ítem 9 & .319 & .223 & .370 & .273 \\
Ítem 10 & .589 & .515 & .629 & .534 \\
Ítem 11 & .448 & .489 & .415 & .489 \\
Ítem 12 & .406 & .291 & .450 & .356 \\
Ítem 13 & .402 & .436 & .373 & .445 \\
Ítem 14 & .441 & .382 & .486 & .385 \\
Ítem 15 & .439 & .360 & .472 & .358 \\
\hline Alfa de Cronbach & .781 & .420 & .606 & .7401 \\
\hline
\end{tabular}

madre fue .804; en ambos casos, alcanzaron un nivel moderado de consistencia interna. Cabe destacar que el procedimiento de análisis de confiabilidad es previo al análisis de validez (Celina \& Campo, 2005), lo que permite para el presente estudio la depuración de un ítem en cada subescala de la Escala de Estilos Parentales.

\section{Evidencias de validez}

A fin de aportar evidencias de la validez de la Escala de Estilos Parentales (MOPS) en sus dos subescalas (padre y madre), se partió del marco teórico postulado por Parker et al. (1997), que plantea la existencia de tres dimensiones: indiferencia, abuso y sobreprotección. Por tanto, se procedió a realizar 
un análisis factorial confirmatorio; sin embargo, en caso de que no se reproduzca el modelo teórico previo, se procederá a realizar un análisis factorial exploratorio.

\section{Análisis factorial confirmatorio}

Se usó la técnica multivariante modelo de ecuaciones estructurales por medio del programa EQS (Bentler, 2006), con el fin de establecer la varianza de las variables latentes en $1.0 \mathrm{y}$ de los términos de error especificados como parámetros libres. Se empleó el método robusto, debido a que la muestra no poseía una distribución normal, $p<$ 0.05 (ver anexo 3). Se utilizaron los siguientes indicadores de bondad de ajuste: (1) chi-cuadrado $\left(X^{2}\right)$; (2) índice de ajuste normado del chi-cuadrado $\left(X^{2} /\right.$ $d f$, relative/normed chi-square); (3) raíz cuadrada media de error de aproximación (RMSEA, Root Mean Square Error of Approximation); (4) índice de ajuste normado (NFI, Normed-Fit Index); (5) índice de ajuste comparativo de Bentler (CFI, Comparative Fit Index). De acuerdo con Hooper, Coughlan y Mullen (2008), se considera un buen ajuste cuando el nivel de significación del valor chi-cuadrado es superior al 0.05 , el valor del índice de ajuste normado del chi-cuadrado oscila entre 2.0 y 5.0 , el RMSEA es inferior a 0.07 y los valores NFI y CFI con superiores al 0.95.

Por lo mencionado, se observa en la tabla 4 que todos los índices de bondad de ajuste resultan ser insatisfactorios en ambas subescalas (padre y madre); por ello, el modelo de tres factores planteado por Parker et al. (1997) resulta inapropiado para el conjunto de 14 ítems.

\section{Análisis factorial exploratorio}

El análisis factorial confirmatorio rechazó la hipótesis teórica donde se afirma que los 14 ítems se agrupan en tres factores, planteada por Parker et al. (1997). En ese sentido, se buscó a través de un análisis factorial exploratorio el

Tabla 4

Índices de ajuste del análisis factorial confirmatorio (modelo de tres factores)

\begin{tabular}{lcccccc}
\hline & Chi-cuadrado $\left(X^{2}\right)$ & $D f$ & $X^{2} / d f$ & RMSEA & NFI & CFI \\
\hline Subescala: Padre & $2,545.0493^{* *}$ & 77 & 33.05 & 0.116 & 0.489 & 0.496 \\
Subescala: Madre & $950.887^{* *}$ & 74 & 12.35 & 0.071 & 0.766 & 0.779 \\
\hline
\end{tabular}

${ }^{* *} p<0.01$ 
Tabla 5

Estimadores para el uso del análisis factorial exploratorio

\begin{tabular}{lccc}
\hline & Determinante & $\begin{array}{c}\text { Medida de adecuación } \\
\text { de muestreo KMO }\end{array}$ & $\begin{array}{c}\text { Test de esfericidad de } \\
\text { Bartlet }\end{array}$ \\
\hline Subescala: Padre & .015 & .918 & $9,942.278^{* *}$ \\
Subescala: Madre & .040 & .878 & $7,592.001^{* *}$ \\
\hline
\end{tabular}

${ }^{* *} p<.01$

Elaboración propia.

Tabla 6

Estructura factorial de la Escala de Estilos Parentales

\begin{tabular}{|c|c|c|c|c|c|c|c|}
\hline \multicolumn{4}{|c|}{ Subescala de estilos parentales del padre } & \multicolumn{4}{|c|}{ Subescala de estilos parentales de la madre } \\
\hline Ítems & Abuso & Sobreprotección & Indiferencia & Ítems & Abuso & Sobreprotección & Indiferencia \\
\hline 2 & .629 & & & 2 & .430 & & \\
\hline 3 & .665 & & & 3 & & .711 & \\
\hline 4 & .593 & & & 4 & .619 & & \\
\hline 5 & .366 & & & 5 & .704 & & \\
\hline 6 & & .334 & & 6 & & .634 & \\
\hline 7 & .526 & & & 7 & .209 & & \\
\hline 8 & & & .813 & 8 & & & .787 \\
\hline 9 & .606 & & & 9 & .505 & & \\
\hline 10 & .355 & & & 10 & .719 & & \\
\hline 11 & & & .331 & 11 & & & .250 \\
\hline 12 & & & .224 & 12 & & & .308 \\
\hline 13 & & & .737 & 13 & & & .804 \\
\hline 14 & & & .387 & 14 & & & .420 \\
\hline 15 & & .429 & & 15 & & .480 & \\
\hline
\end{tabular}

mejor modelo explicativo de la Escala de Estilos Parentales.

Para considerar lo apropiado del análisis factorial exploratorio, se analizó el valor del determinante, la medida de adecuación de muestreo KMO y el test de esfericidad de Bartlet. Como se aprecia en la tabla 5, para ambas subescalas el determinante se aproxima a 0.0 , la medida de adecuación de muestreo KMO es próxima a 1.0, y el test de esfericidad de Bartlet es muy significativo. 
Tabla 7

Correlaciones entre la subescala de estilos parentales del padre y sus factores

\begin{tabular}{lccccc}
\hline & \multicolumn{2}{c}{$\begin{array}{c}\text { Subescala } \\
\text { de estilos } \\
\text { parentales } \\
\text { del padre }\end{array}$} & Abuso & Sobreprotección & Indiferencia \\
\hline $\begin{array}{l}\text { Subescala de } \\
\text { estilos parentales } \\
\text { del padre }\end{array}$ & Sig. (bilateral) & 1.000 & $.890^{* *}$ & $.746^{* *}$ & $.703^{* *}$ \\
& d de Cohen & .000 & .000 & .000 \\
Abuso & Rho & 2.8 & 2.2 & 2 \\
& Sig. (bilateral) & 1,000 & $.550^{* *}$ & $.405^{* *}$ \\
& d de Cohen & & .000 & .000 \\
Sobreprotección & Rho & & 1.5 & 0.9 \\
& Sig. (bilateral) & & 1.000 & $.501^{* *}$ \\
& d de Cohen & & & .000 \\
\hline
\end{tabular}

** La correlación es significativa al nivel 0.01 (bilateral).

Con lo anterior se procedió a realizar el análisis factorial exploratorio aplicándose la técnica de los componentes principales y con rotación varimax.

En el análisis factorial exploratorio de ejes principales, se empleó el método de extracción de factores que expliquen aproximadamente el $50 \%$ de la varianza total. De esta manera, se encontró el modelo más apropiado de tres factores que explicaron en la subescala del padre el $53,819 \%$ y en la subescala de la madre el 49,260 \% (ver anexo 2). A su vez, el método de rotación fue ortogonal de tipo varimax.

Finalmente, se obtuvo una estructura factorial de la Escala de Estilos Parenta- les para la muestra peruana, que aparece en la tabla 6. Allí se indican los factores con sus ítems correspondientes y las saturaciones o cargas de estos. Estas saturaciones, por ser en casi todos los casos de .50 o más, propician la clara definición e interpretación de los factores (Comrey, 1985; citado en Aliaga et al., 2006).

Posteriormente, se realizó un análisis de la correlación entre cada subescala de estilos parentales del padre y de la madre, con los tres factores obtenidos, mediante la prueba no paramétrica rho de Spearman. Se observa, en la tabla 7, correlaciones directas, altas y muy significativas entre la subescala de estilos parentales del padre y sus factores. De igual modo, se aprecia 
Tabla 8

Correlaciones entre la subescala de estilos parentales de la madre y sus factores

\begin{tabular}{|c|c|c|c|c|c|}
\hline & & $\begin{array}{c}\text { Subescala } \\
\text { de estilos } \\
\text { parentales } \\
\text { de la madre }\end{array}$ & Abuso & Sobreprotección & Indiferencia \\
\hline \multirow{3}{*}{$\begin{array}{l}\text { Subescala de } \\
\text { estilos parentales } \\
\text { de la madre }\end{array}$} & Rho & 1.000 & $.747^{* *}$ & $.843^{* *}$ & $.606^{\star *}$ \\
\hline & Sig. (bilateral) & & .000 & .000 & .000 \\
\hline & $\mathrm{d}$ de Cohen & & 2.2 & 2.6 & 1.5 \\
\hline \multirow{3}{*}{ Abuso } & Rho & & 1.000 & $.499^{* *}$ & $.374^{* *}$ \\
\hline & Sig. (bilateral) & & & .000 & .000 \\
\hline & $\mathrm{d}$ de Cohen & & & 1.1 & 0.8 \\
\hline \multirow{3}{*}{ Sobreprotección } & Rho & & & 1,000 & $.242^{* *}$ \\
\hline & Sig. (bilateral) & & & &, 000 \\
\hline & $\mathrm{d}$ de Cohen & & & & 0.5 \\
\hline
\end{tabular}

**La correlación es significativa al nivel .01 (bilateral).

correlaciones directas, altas y muy significativas entre los diferentes factores.

Del mismo modo, se aprecia, en la tabla 8, correlaciones directas, altas y muy significativas entre la subescala de estilos parentales de la madre y sus factores. Asimismo, se obtuvo correlaciones directas, medias y muy significativas entre los factores de la subescala de estilos parentales de la madre.

\section{Elaboración de baremos}

Los baremos presentados en el anexo 5 han sido elaborados en puntuaciones equivalentes de tipo percentilar, con base en el puntaje directo inicial. Estas puntua- ciones equivalentes corresponden a los baremos normativos en percentiles (Pc); las tablas se han distribuido de acuerdo a cada subescala por región geográfica: costa, sierra y selva, con el objetivo de brindar perfiles diferenciales que no estén afectados por factores sociales y culturales. Cabe señalar que en las comparaciones según sexo y región geográfica solo se detectó diferencias significativas según la región geográfica (ver anexo 4), por lo que se hace conveniente el uso diferencial de baremos por región geográfica.

\section{DISCUSIÓN DE RESULTADOS}

El presente estudio fue desarrollado en una muestra total de 2370 adolescen- 
tes de 14 ciudades representativas de Perú, con edades entre 13 a 19 años. Los resultados obtenidos validan las propiedades psicométricas de fiabilidad con un alfa de Cronbach de la subescala de estilos parentales del padre de .853 y en la subescala de estilos parentales de la madre con .804, de la Escala de Estilos Parentales (MOPS) de Parker et al. (1997) en nuestro medio.

La escala original se encuentra en inglés; sin embargo, se elaboró una adaptación lingüística para nuestro medio: se tradujo al español y se eliminó el primer ítem de la escala, por lo que quedaron en total 14 ítems.

En el proceso de análisis de los datos se procedió, en un primer momento, a realizar un análisis factorial confirmatorio; sin embargo, este resultó insatisfactorio, debido a que no se reprodujo el modelo de los tres factores propuesto por Paker et al. (1997), definidos como indiferencia, abuso y sobreprotección, los cuales son considerados por los autores como estilos parentales disfuncionales. En un segundo momento, se procedió a realizar un análisis factorial exploratorio, mediante el cual se logró reproducir los tres factores en la Escala de Estilos Parentales Disfuncionales (MOPS).

No obstante, los tres factores obtenidos difieren en la reagrupación de algunos ítems, sobre todo en la distribución de los ítems en las subescalas propuestas por los autores Paker et al. (1997); los ítems difieren según las figuras parentales (padre, madre). Dichas diferencias responden a factores contextuales y de género, ya que tras lo analizado se apreció que ítems que en la subescala de estilos parentales disfuncionales de la madre miden la sobreprotección, en la subescala de estilos parentales disfuncionales del padre miden el abuso, tal es el caso del ítem "Me controlaba demasiado". Las variaciones entre las subescalas según las diferencia de género responderían a que los cuidados de excesivo control por parte de la figura paterna son percibidos en los adolescentes como abuso, mas no como sobreprotección. Dichas diferencias estarían relacionadas con factores culturales y roles sociales existentes en nuestro país.

\section{Conclusiones}

1. La Escala de Estilos Parentales Disfuncionales (MOPS) ha demostrado confiabilidad en la medición por los métodos de consistencia interna por la fórmula de Cronbach y por el método de correlación ítem-test.

2. En el análisis factorial exploratorio se obtuvieron tres factores: indiferencia, abuso y sobreprotección, los cuales coinciden con la teoría subyacente a la prueba: indiferencia, abuso y sobreprotección.

3. En síntesis, la prueba tiene validez y confiabilidad estadística, lo que hace posible su empleo en estudiantes de secundaria del Perú. 


\section{REFERENCIAS}

Aliaga, J., Rodríguez, L., Ponce, C., Frisancho, A., \& Enríquez, J. (2006). Escala de Desesperanza de Beck (BHS): Adaptación y características psicométricas. Revista de Investigación en Psicología, 9(1), 69-79.

Baumrind, D. (1996). The Discipline Controversy Revisited. Family Relations, 45, 4, 405-414.

Baumrind, D. (2005). Patterns of Parental Authority and Adolescent $\mathrm{Au}-$ tonomy. En J. Smetana (Ed.), New Directions for Child Development: Changes in Parental Authority during Adolescence (pp. 61-69). San Francisco, CA: Jossey-Bass.

Bedoya, E. (2012). Apego y esquizotipia en población no clínica. (Tesis doctoral). Universidad Autónoma de Barcelona. Bellaterra, España.

Bentler, P. (2006). EQS 6 Structural Equations Program Manual. Encino, CA: Multivariate Software, Inc.

Bowlby, J. (1969). Attachment and Loss (volumen 1). Nueva York: Basic Books.

Bowlby, J. (1973). Attachment and Loss (volumen 3. Loss: Sadness and depression). Nueva York: Basic Books.

Bowlby, J. (1977). The Making and Breaking of Affectional Bonds: I. A etiology and psychopathology in the light of attachment theory. British Journal of Psychiatry, 130, 201-210.
Celina, H. \& Campo, A. (2005). Aproximación al uso del coeficiente alfa de Cronbach. Revista Colombiana de Psiquiatría, 34(4), 572-580.

Estévez, A., \& Calvete, E. (2007). Esquemas cognitivos en personas con conducta de juego patológico y su relación con experiencias de crianza. Clínica y Salud, 18(1), 23-43.

Estévez, E., Murgui, S., Musitu, G., \& Moreno, D. (2008). Adolescent Aggression: Effects of Gender and Family and School Environments. Journal of Adolescence, 31, 433-450.

Fourment, K. (2009). Validez y confiabilidad del auto cuestionario de modelos internos de relaciones de apego (CaMir) en un grupo de madres de Lima Metropolitana. (Tesis de licenciatura). Pontificia Universidad Católica del Perú. Lima, Perú.

Hogan, T. (2004). Pruebas psicológicas. México D. F.: Manual Moderno.

Hooper, D., Coughlan, J., \& Mullen, M. (2008). Structural Equation Modelling: Guidelines for Determining Model Fit. Electronic Journal of Business Research Methods, 6(1), 53-60.

Kerlinger, F., \& Lee, H. (2002). Investigación del comportamiento. (4. ${ }^{\mathrm{a}} \mathrm{ed}$.). México D. F.: McGraw-Hill.

Melis, F., Dávila, M. Á., Ormeño, V., Vera, V., Greppi, C., \& Gloger, S. (2001). Estandarización del PBI (Pa- 
rental Bonding Instrument), versión adaptada a la población entre 16 y 64 años del Gran Santiago. Revista Chilena de Neuro-Psiquiatría, 39(2), 132-139.

Parker, G., Roussos, J., Hadzi-Pavlovic, D., Mitchell, P., Wilhelm, K. \& Austin, M. P. (1997). The development of a refined measure of dysfunctional parenting and assessment of its relevance in patients with affective disorders. Psychological Medicine, 27(5), 1193-1203, doi: 10.1017/ S003329179700545X.

Parker, G., Tupling, H., \& Brown, L. (1979). A Parental Bonding Instruments. British Journal of Medical Psychology, 37(52), 1-10.

Raya, A. (2008). Estudio sobre los estilos educativos parentales y su relación con los trastornos de la conducta en la infancia (Tesis doctoral). Universidad de Córdoba. Córdoba, España.

Sánchez-Queija, I. (2007). Análisis longitudinal de las relaciones con los iguales durante la adolescencia. (Tesis doctoral no publicada). Universidad de Sevilla. Sevilla, España.

Sánchez, H., \& Reyes, C. (2009). Metodología y diseños en la investigación cientifica. (4. ${ }^{\mathrm{a}}$ ed.). Lima: Editorial Visión Universitaria.

Steinber, L., Blatt-Eisengart, I., \& Cauffman, E. (2006). Patterns of competence and adjustment among adolescents from authoritative, authoritarian, indulgent and 27 neglectful homes: a replication in a simple of serious juvenile offenders. Journal of Research on Adolescence, 16(1), 47-58. 


\section{ANEXO 1}

\section{Tabla 9}

Estadísticos ítems-test de la subescala de estilos parentales del padre

\begin{tabular}{|c|c|c|c|c|}
\hline & $\begin{array}{l}\text { Media de la } \\
\text { escala si se } \\
\text { elimina el } \\
\text { elemento }\end{array}$ & $\begin{array}{c}\text { Varianza de } \\
\text { la escala si } \\
\text { se elimina el } \\
\text { elemento }\end{array}$ & $\begin{array}{c}\text { Correlación } \\
\text { elemento-total } \\
\text { corregida }\end{array}$ & $\begin{array}{l}\text { Alfa de Cronbach } \\
\text { si se elimina el } \\
\text { elemento }\end{array}$ \\
\hline Ítem 1 & 8.19 & 45.098 & .007 & .853 \\
\hline Ítem 2 & 9.23 & 41.404 & .508 & .816 \\
\hline Ítem 3 & 8.32 & 41.601 & .294 & .831 \\
\hline Ítem 4 & 9.26 & 40.649 & .563 & .813 \\
\hline Ítem 5 & 9.23 & 40.364 & .588 & .811 \\
\hline Ítem 6 & 9.01 & 40.766 & .511 & .815 \\
\hline Ítem 7 & 8.85 & 40.707 & .415 & .821 \\
\hline Ítem 8 & 8.91 & 40.200 & .398 & .823 \\
\hline Ítem 9 & 9.24 & 40.381 & .585 & .812 \\
\hline Ítem 10 & 9.33 & 40.593 & .635 & .810 \\
\hline Ítem 11 & 8.73 & 39.658 & .467 & .818 \\
\hline Ítem 12 & 9.26 & 40.558 & .528 & .814 \\
\hline Ítem 13 & 9.04 & 38.875 & .528 & .813 \\
\hline Ítem 14 & 9.39 & 41.858 & .465 & .819 \\
\hline Ítem 15 & 9.11 & 39.464 & .599 & .809 \\
\hline
\end{tabular}


Tabla 10

Estadísticos ítems-test de la subescala de estilos parentales de la madre

\begin{tabular}{|c|c|c|c|c|}
\hline & $\begin{array}{c}\text { Media de la } \\
\text { escala si se } \\
\text { elimina el } \\
\text { elemento }\end{array}$ & $\begin{array}{l}\text { Varianza de } \\
\text { la escala si } \\
\text { se elimina el } \\
\text { elemento }\end{array}$ & $\begin{array}{l}\text { Correlación } \\
\text { elemento-total } \\
\text { corregida }\end{array}$ & $\begin{array}{l}\text { Alfa de Cronbach } \\
\text { si se elimina el } \\
\text { elemento }\end{array}$ \\
\hline Ítem 1 & 7.90 & 34.106 & .063 & .804 \\
\hline Ítem 2 & 9.28 & 31.910 & .448 & .765 \\
\hline Ítem 3 & 8.18 & 31.855 & .277 & .781 \\
\hline Ítem 4 & 9.33 & 31.707 & .467 & .764 \\
\hline Ítem 5 & 9.42 & 32.007 & .511 & .762 \\
\hline Ítem 6 & 9.00 & 30.733 & .495 & .760 \\
\hline Ítem 7 & 8.94 & 31.749 & .332 & .775 \\
\hline Ítem 8 & 9.01 & 31.127 & .319 & .778 \\
\hline Ítem 9 & 9.40 & 31.151 & .589 & .756 \\
\hline Ítem 10 & 9.51 & 32.716 & .448 & .767 \\
\hline Ítem 11 & 8.90 & 30.984 & .406 & .768 \\
\hline Ítem 12 & 9.52 & 33.031 & .402 & .770 \\
\hline Ítem 13 & 9.26 & 30.706 & .441 & .764 \\
\hline Ítem 14 & 9.53 & 32.665 & .439 & .767 \\
\hline Ítem 15 & 9.28 & 30.822 & .551 & .756 \\
\hline
\end{tabular}




\section{ANEXO 2}

\section{Tabla 11}

Varianza total explicada de la subescala de estilos parentales del padre con 14 ítems

\begin{tabular}{|c|c|c|c|c|c|c|c|c|c|}
\hline \multirow{2}{*}{ Factor } & \multicolumn{3}{|c|}{ Autovalores iniciales } & \multicolumn{3}{|c|}{$\begin{array}{l}\text { Sumas de las saturaciones al } \\
\text { cuadrado de la extracción }\end{array}$} & \multicolumn{3}{|c|}{$\begin{array}{l}\text { Suma de las saturaciones al } \\
\text { cuadrado de la rotación }\end{array}$} \\
\hline & Total & $\begin{array}{c}\% \text { de la } \\
\text { varianza }\end{array}$ & $\begin{array}{c}\% \\
\text { acumulado }\end{array}$ & Total & $\begin{array}{c}\% \text { de la } \\
\text { varianza }\end{array}$ & $\begin{array}{c}\% \\
\text { acumulado }\end{array}$ & Total & $\begin{array}{c}\% \text { de la } \\
\text { varianza }\end{array}$ & $\begin{array}{c}\% \\
\text { acumulado }\end{array}$ \\
\hline 1 & 5.217 & 37.264 & 37.264 & 5.217 & 37.264 & 37.264 & 2.937 & 20.978 & 20.978 \\
\hline 2 & 1.279 & 9.132 & 46.396 & 1.279 & 9.132 & 46.396 & 2.899 & 20.709 & 41.687 \\
\hline 3 & 1.042 & 7.446 & 53.842 & 1.042 & 7.446 & 53.842 & 1.702 & 12.155 & 53.842 \\
\hline 4 & .806 & 5.756 & 59.598 & & & & & & \\
\hline 5 & .776 & 5.546 & 65.144 & & & & & & \\
\hline 6 & .725 & 5.180 & 70.324 & & & & & & \\
\hline 7 & .692 & 4.942 & 75.266 & & & & & & \\
\hline 8 & .599 & 4.277 & 79.544 & & & & & & \\
\hline 9 & .560 & 3.999 & 83.543 & & & & & & \\
\hline 10 & .528 & 3.770 & 87.313 & & & & & & \\
\hline 11 & .489 & 3.495 & 90.808 & & & & & & \\
\hline 12 & .470 & 3.354 & 94.162 & & & & & & \\
\hline 13 & .412 & 2.942 & 97.104 & & & & & & \\
\hline 14 & .405 & 2.896 & 100.000 & & & & & & \\
\hline
\end{tabular}

Método de extracción: análisis de componentes principales. 


\section{ANEXO 3}

\section{Tabla 12}

Varianza total explicada de la subescala de estilos parentales de la madre con 14 ítems

\begin{tabular}{|c|c|c|c|c|c|c|c|c|c|}
\hline \multirow{2}{*}{ Factor- } & \multicolumn{3}{|c|}{ Autovalores iniciales } & \multicolumn{3}{|c|}{$\begin{array}{l}\text { Sumas de las saturaciones } \\
\text { al cuadrado de la extracción }\end{array}$} & \multicolumn{3}{|c|}{$\begin{array}{l}\text { Suma de las saturaciones al } \\
\text { cuadrado de la rotación }\end{array}$} \\
\hline & Total & $\begin{array}{c}\% \text { de la } \\
\text { varianza }\end{array}$ & $\begin{array}{c}\% \\
\text { acumulado }\end{array}$ & Total & $\begin{array}{c}\% \text { de la } \\
\text { varianza }\end{array}$ & $\begin{array}{c}\% \\
\text { acumulado }\end{array}$ & Total & $\begin{array}{c}\% \text { de la } \\
\text { varianza }\end{array}$ & $\begin{array}{c}\% \\
\text { acumulado }\end{array}$ \\
\hline 1 & 4.397 & 31.409 & 31.409 & 4.397 & 31.409 & 31.409 & 2.786 & 19.897 & 19.897 \\
\hline 2 & 1.346 & 9.616 & 41.025 & 1.346 & 9.616 & 41.025 & 2.262 & 16.157 & 36.054 \\
\hline 3 & 1.153 & 8.235 & 49.260 & 1.153 & 8.235 & 49.260 & 1.849 & 13.206 & 49.260 \\
\hline 4 & .963 & 6.878 & 56.137 & & & & & & \\
\hline 5 & .837 & 5.977 & 62.114 & & & & & & \\
\hline 6 & .795 & 5.678 & 67.792 & & & & & & \\
\hline 7 & .761 & 5.433 & 73.225 & & & & & & \\
\hline 8 & .644 & 4.598 & 77.823 & & & & & & \\
\hline 9 & .581 & 4.150 & 81.973 & & & & & & \\
\hline 10 & .556 & 3.974 & 85.947 & & & & & & \\
\hline 11 & .527 & 3.766 & 89.713 & & & & & & \\
\hline 12 & .505 & 3.610 & 93.323 & & & & & & \\
\hline 13 & .469 & 3.351 & 96.674 & & & & & & \\
\hline 14 & .466 & 3.326 & 100.000 & & & & & & \\
\hline
\end{tabular}

Método de extracción: análisis de componentes principales. 


\section{ANEXO 4}

Tabla 13

Normalidad de los 14 ítems de la subescala de estilos parentales del padre

\begin{tabular}{cccc}
\hline & \multicolumn{2}{c}{ Parámetros } & \\
\cline { 2 - 3 } Ítems & Media & $\begin{array}{c}\text { Desviación } \\
\text { Estándar }\end{array}$ & Kolmogorov-Smirnov Z \\
\hline Ítem 2 & .42 & .677 & $19.327^{\text {** }}$ \\
Ítem 3 & 1.33 & .982 & $12.561^{\text {** }}$ \\
Ítem 4 & .39 & .714 & $20.413^{* *}$ \\
Ítem 5 & .42 & .722 & $19.965^{\text {** }}$ \\
Ítem 6 & .64 & .758 & $14.366^{* *}$ \\
Ítem 7 & .80 & .900 & $12.971^{* *}$ \\
Ítem 8 & .74 & 1.006 & $15.987^{* *}$ \\
Ítem 9 & .41 & .723 & $20.410^{* *}$ \\
Ítem 10 & .32 & .651 & $21.779^{* *}$ \\
Ítem 11 & .92 & .968 & $12.670^{* *}$ \\
Ítem 12 & .39 & .764 & $21.370^{* *}$ \\
Ítem 13 & .61 & .979 & $18.941^{* *}$ \\
Ítem 14 & .26 & .662 & $23.667^{* *}$ \\
Ítem 15 & .54 & .818 & $17.867^{* *}$ \\
\hline
\end{tabular}

${ }^{* *} p<.01$ 
Tabla 14

Normalidad de los 14 ítems de la subescala de estilos parentales de la madre

\begin{tabular}{|c|c|c|c|}
\hline \multirow[b]{2}{*}{ Ítems } & \multicolumn{2}{|c|}{ Parámetros } & \multirow[b]{2}{*}{ Kolmogorov-Smirnov Z } \\
\hline & Media & $\begin{array}{l}\text { Desviación } \\
\text { Estándar }\end{array}$ & \\
\hline Ítem 2 & .47 & .703 & $18.638^{* *}$ \\
\hline Ítem 3 & 1.57 & .997 & $12.723^{\star *}$ \\
\hline Ítem 4 & .42 & .713 & $19.815^{\star *}$ \\
\hline Ítem 5 & .33 & .618 & $21.241^{* *}$ \\
\hline Ítem 6 & .74 & .828 & $13.601^{* *}$ \\
\hline Ítem 7 & .81 & .906 & $12.907^{\star *}$ \\
\hline Ítem 8 & .74 & 1.049 & $17.094^{* *}$ \\
\hline Ítem 9 & .35 & .665 & $21.516^{\star *}$ \\
\hline Ítem 10 & .24 & .570 & $23.367^{* *}$ \\
\hline Ítem 11 & .84 & .916 & $12.285^{\star *}$ \\
\hline Ítem 12 & .22 & .566 & $23.692^{* *}$ \\
\hline Ítem 13 & .49 & .907 & $20.764^{* *}$ \\
\hline Ítem 14 & .21 & .589 & $24.049^{* *}$ \\
\hline Ítem 15 & .47 & .749 & $18.857^{* *}$ \\
\hline
\end{tabular}

${ }^{* *} p<.01$ 


\section{ANEXO 5}

\section{Tabla 15}

Prueba de U de Mann-Whitney para estimar si existen diferencias de las variables. Estilos parentales y adicción a la internet según sexo

\begin{tabular}{|c|c|c|c|c|c|c|}
\hline Variable & Grupo & $\mathbf{N}$ & $\begin{array}{c}\text { Rango } \\
\text { promedio }\end{array}$ & $\begin{array}{l}\text { U de Mann- } \\
\text { Whitney }\end{array}$ & $\mathbf{z}$ & $\begin{array}{c}\text { Delta de } \\
\text { Cliff }\end{array}$ \\
\hline \multirow{2}{*}{$\begin{array}{l}\text { Estilos parentales } \\
\text { del padre }\end{array}$} & Masculino & 1127 & 1193.78 & \multirow[b]{2}{*}{689.920} & \multirow[b]{2}{*}{-0.596} & \multirow[b]{2}{*}{0.01} \\
\hline & Femenino & 1243 & 1177.04 & & & \\
\hline \multirow{3}{*}{ Indiferencia } & Masculino & 1127 & 1193.12 & \multirow{3}{*}{691.847} & \multirow{3}{*}{-0.521} & \multirow{3}{*}{0.01} \\
\hline & & & & & & \\
\hline & Femenino & 1243 & 1178.59 & & & \\
\hline \multirow{3}{*}{ Abuso } & Masculino & 1127 & 1217.81 & \multirow{3}{*}{662.861} & \multirow{3}{*}{$-2.259^{*}$} & \multirow{3}{*}{0.05} \\
\hline & & & & & & \\
\hline & Femenino & 1243 & 1155.28 & & & \\
\hline \multirow{3}{*}{ Sobreprotección } & Masculino & 1127 & 1192.27 & \multirow{3}{*}{692.805} & \multirow{3}{*}{-0.463} & \multirow{3}{*}{0.01} \\
\hline & & & & & & \\
\hline & Femenino & 1243 & 1179.36 & & & \\
\hline \multirow{2}{*}{$\begin{array}{l}\text { Estilos parentales } \\
\text { de la madre }\end{array}$} & Masculino & 1127 & 1159.19 & \multirow{2}{*}{670.785} & \multirow{2}{*}{-1.786} & \multirow{2}{*}{0.04} \\
\hline & Femenino & 1243 & 1209.35 & & & \\
\hline \multirow{3}{*}{ Indiferencia } & Masculino & 1127 & 1167.88 & \multirow{3}{*}{680.568} & \multirow{3}{*}{-1.209} & \multirow{3}{*}{0.02} \\
\hline & & & & & & \\
\hline & Femenino & 1243 & 1201.48 & & & \\
\hline \multirow{3}{*}{ Abuso } & Masculino & 1127 & 1171.76 & \multirow{3}{*}{684.946} & \multirow{3}{*}{-0.948} & \multirow{3}{*}{0.02} \\
\hline & & & & & & \\
\hline & Femenino & 1243 & 1197.96 & & & \\
\hline \multirow{3}{*}{ Sobreprotección } & Masculino & 1127 & 1159.15 & \multirow{3}{*}{670.730} & \multirow{3}{*}{-1.801} & \multirow{3}{*}{0.04} \\
\hline & & & & & & \\
\hline & Femenino & 1243 & 1209.39 & & & \\
\hline
\end{tabular}

${ }^{*} p<.05$ 


\section{ANEXO 6}

Tabla 16

Prueba de Kruskal-Wallis de muestras independientes para estimar si existen diferencias de las subescalas de estilos parentales del padre y de la madre, según región geográfica

\begin{tabular}{|c|c|c|c|c|c|c|}
\hline Variable & Grupo & $\mathbf{N}$ & $\begin{array}{c}\text { Rango } \\
\text { promedio }\end{array}$ & $\begin{array}{l}\text { Chi- } \\
\text { cuadrado }\end{array}$ & gl & $\begin{array}{l}\text { Delta de } \\
\text { Cliff }\end{array}$ \\
\hline \multirow{3}{*}{$\begin{array}{l}\text { Estilos parentales } \\
\text { del padre }\end{array}$} & Costa & 662 & 1077.24 & \multirow{3}{*}{$40.976^{* *}$} & \multirow{3}{*}{2} & \multirow{3}{*}{0.13} \\
\hline & Sierra & 1216 & 1271.44 & & & \\
\hline & Selva & 492 & 1116.21 & & & \\
\hline \multirow{3}{*}{ Indiferencia } & Costa & 662 & 1090.15 & \multirow{3}{*}{$29.067^{* *}$} & \multirow{3}{*}{2} & \multirow{3}{*}{0.11} \\
\hline & Sierra & 1216 & 1256.81 & & & \\
\hline & Selva & 492 & 1137.54 & & & \\
\hline \multirow{3}{*}{ Abuso } & Costa & 662 & 1080.47 & \multirow{3}{*}{$58.061^{* *}$} & \multirow{3}{*}{2} & \multirow{3}{*}{0.16} \\
\hline & Sierra & 1216 & 1287.55 & & & \\
\hline & Selva & 492 & 1071.97 & & & \\
\hline \multirow{3}{*}{ Sobreprotección } & Costa & 662 & 1105.97 & \multirow{3}{*}{$25.317^{* \star}$} & \multirow{3}{*}{2} & \multirow{3}{*}{0.10} \\
\hline & Sierra & 1216 & 1253.42 & & & \\
\hline & Selva & 492 & 1124.64 & & & \\
\hline \multirow{3}{*}{$\begin{array}{l}\text { Estilos parentales } \\
\text { de la madre }\end{array}$} & Costa & 662 & 1095.36 & \multirow{3}{*}{$35.259^{\star *}$} & \multirow{3}{*}{2} & \multirow{3}{*}{0.12} \\
\hline & Sierra & 1216 & 1266.55 & & & \\
\hline & Selva & 492 & 1106.46 & & & \\
\hline \multirow{3}{*}{ Indiferencia } & Costa & 662 & 1039.72 & \multirow{3}{*}{$57.706^{\star *}$} & \multirow{3}{*}{2} & \multirow{3}{*}{0.16} \\
\hline & Sierra & 1216 & 1282.27 & & & \\
\hline & Selva & 492 & 1142.49 & & & \\
\hline \multirow{3}{*}{ Abuso } & Costa & 662 & 1150.88 & \multirow{3}{*}{$19.933^{* *}$} & \multirow{3}{*}{2} & \multirow{3}{*}{0.09} \\
\hline & Sierra & 1216 & 1242.18 & & & \\
\hline & Selva & 492 & 1091.99 & & & \\
\hline \multirow{3}{*}{ Sobreprotección } & Costa & 662 & 1182.65 & \multirow{3}{*}{$8.711^{*}$} & \multirow{3}{*}{2} & \multirow{3}{*}{0.06} \\
\hline & Sierra & 1216 & 1217.38 & & & \\
\hline & Selva & 492 & 1110.54 & & & \\
\hline
\end{tabular}

${ }^{*} p<.05,{ }^{* *} p<.01$ 


\section{ANEXO 7}

\section{Tabla 17}

\section{Baremos generales de la Escala de Estilos Parentales}

\begin{tabular}{|c|c|c|c|c|c|c|c|c|c|}
\hline \multirow{2}{*}{$\begin{array}{l}\text { Percenti- } \\
\text { les }\end{array}$} & \multicolumn{4}{|c|}{$\begin{array}{l}\text { Puntajes directos: subescala de } \\
\text { los estilos parentales del padre }\end{array}$} & \multicolumn{4}{|c|}{$\begin{array}{l}\text { Puntajes directos: subescala de } \\
\text { los estilos parentales de la madre }\end{array}$} & \multirow{2}{*}{$\begin{array}{l}\text { Categoría } \\
\text { diagnóstica }\end{array}$} \\
\hline & $A b$ & So & In & ET & $A b$ & So & In & ET & \\
\hline 99 & $14-21$ & 6 & $13-15$ & $25-42$ & $12-18$ & $7-9$ & $11-15$ & $28-42$ & Alto \\
\hline 95 & $11-13$ & $4-5$ & $9-12$ & $21-24$ & 8-11 & --- & $8-10$ & $22-27$ & Alto \\
\hline 90 & $8-10$ & 3 & $7-8$ & $16-20$ & 7 & 6 & $6-7$ & $16-21$ & Alto \\
\hline 85 & 7 & --- & 6 & $14-15$ & $5-6$ & 5 & 5 & $13-15$ & Medio \\
\hline 80 & --- & --- & 5 & $12-13$ & --- & --- & --- & $11-12$ & Medio \\
\hline 75 & 6 & --- & --- & 11 & 4 & 4 & 4 & 10 & Medio \\
\hline 70 & --- & 2 & 4 & 10 & --- & --- & --- & 9 & Medio \\
\hline 65 & 5 & --- & --- & 9 & 3 & --- & 3 & --- & Medio \\
\hline 60 & --- & --- & 3 & & --- & --- & --- & 8 & Medio \\
\hline 55 & 4 & --- & --- & 8 & --- & 3 & --- & --- & Medio \\
\hline 50 & 3 & 1 & 2 & 7 & 2 & 2 & 2 & 7 & Medio \\
\hline 45 & --- & --- & --- & 6 & 1 & --- & 1 & 6 & Medio \\
\hline 40 & --- & --- & 1 & & --- & --- & --- & --- & Medio \\
\hline 35 & --- & 0 & --- & 5 & --- & --- & --- & 5 & Medio \\
\hline 30 & 2 & --- & --- & & --- & --- & --- & 4 & Medio \\
\hline 25 & --- & --- & --- & 4 & --- & 1 & --- & --- & Medio \\
\hline 20 & --- & --- & 0 & & 0 & --- & 0 & 3 & Medio \\
\hline 15 & 1 & --- & --- & 3 & --- & --- & --- & --- & Medio \\
\hline 10 & --- & --- & --- & 2 & --- & --- & --- & 2 & Bajo \\
\hline 5 & --- & --- & --- & 1 & --- & 0 & --- & 1 & Bajo \\
\hline 1 & 0 & --- & --- & 0 & --- & --- & --- & 0 & Bajo \\
\hline $\mathrm{N}$ & 2370 & 2370 & 2370 & 2370 & 2370 & 2370 & 2370 & 2370 & $\mathrm{~N}$ \\
\hline Media & 4.18 & 1.18 & 2.92 & 8.28 & 2.61 & 2.78 & 2.51 & 7.90 & Media \\
\hline $\begin{array}{l}\text { Des- } \\
\text { viación } \\
\text { estándar }\end{array}$ & 3.096 & 1.316 & 2.987 & 5.715 & 2.743 & 1.859 & 2.604 & 5.840 & $\begin{array}{l}\text { Desviación } \\
\text { estándar }\end{array}$ \\
\hline Mínimo & 0 & 0 & 0 & 0 & 0 & 0 & 0 & 0 & Mínimo \\
\hline Máximo & 21 & 6 & 15 & 35 & 18 & 9 & 15 & 37 & Máximo \\
\hline
\end{tabular}


Tabla 18

Baremos de la Escala de Estilos Parentales para la costa (Tacna, Lima, Trujillo y Tumbes)

\begin{tabular}{|c|c|c|c|c|c|c|c|c|c|}
\hline \multirow{2}{*}{$\begin{array}{l}\text { Percenti- } \\
\text { les }\end{array}$} & \multicolumn{4}{|c|}{$\begin{array}{l}\text { Puntajes directos: subescala de } \\
\text { los estilos parentales del padre }\end{array}$} & \multicolumn{4}{|c|}{$\begin{array}{l}\text { Puntajes directos: subescala de } \\
\text { los estilos parentales de la madre }\end{array}$} & \multirow{2}{*}{$\begin{array}{l}\text { Categoría } \\
\text { diagnóstica }\end{array}$} \\
\hline & $A b$ & So & In & ET & $A b$ & So & In & ET & \\
\hline 99 & $14-21$ & $5-6$ & $13-15$ & $27-42$ & $11-18$ & $8-9$ & $9-15$ & $24-42$ & Alto \\
\hline 95 & $9-13$ & 4 & $8-12$ & $19-26$ & 8-10 & $6-7$ & $6-8$ & $17-23$ & Alto \\
\hline 90 & $7-8$ & 3 & $6-7$ & $15-18$ & $6-7$ & 5 & --- & $13-16$ & Alto \\
\hline 85 & --- & --- & 5 & $12-14$ & 5 & --- & $4-5$ & $11-12$ & Medio \\
\hline 80 & 6 & --- & --- & $10-11$ & --- & --- & --- & 10 & Medio \\
\hline 75 & 5 & 2 & 4 & --- & 4 & 4 & 3 & 9 & Medio \\
\hline 70 & --- & --- & --- & 9 & --- & --- & --- & --- & Medio \\
\hline 65 & --- & --- & 3 & 8 & 3 & --- & --- & 8 & Medio \\
\hline 60 & 4 & --- & --- & 7 & --- & --- & 2 & --- & Medio \\
\hline 55 & --- & --- & --- & --- & --- & 3 & --- & 7 & Medio \\
\hline 50 & 3 & 1 & 2 & 6 & 2 & 2 & 1 & 6 & Medio \\
\hline 45 & --- & --- & 1 & 5 & & --- & --- & 5 & Medio \\
\hline 40 & 2 & --- & --- & --- & 1 & --- & --- & --- & Medio \\
\hline 35 & --- & 0 & --- & 4 & --- & --- & --- & --- & Medio \\
\hline 30 & --- & --- & --- & --- & --- & --- & --- & 4 & Medio \\
\hline 25 & --- & --- & 0 & 3 & --- & 1 & 0 & --- & Medio \\
\hline 20 & 1 & --- & --- & --- & --- & --- & --- & 3 & Medio \\
\hline 15 & --- & --- & --- & 2 & 0 & --- & --- & --- & Medio \\
\hline 10 & --- & --- & --- & 1 & --- & --- & --- & 2 & Bajo \\
\hline 5 & --- & --- & --- & 0 & --- & 0 & --- & 1 & Bajo \\
\hline 1 & --- & --- & --- & --- & --- & --- & --- & 0 & Bajo \\
\hline $\mathrm{N}$ & 662 & 662 & 662 & 662 & 662 & 662 & 662 & 662 & $\mathrm{~N}$ \\
\hline Media & 3.60 & 1.12 & 2.44 & 7.15 & 2.53 & 2.66 & 1.86 & 7.05 & Media \\
\hline $\begin{array}{l}\text { Des- } \\
\text { viación } \\
\text { estándar }\end{array}$ & 3.023 & 1.247 & 2.744 & 5.897 & 2.501 & 1.646 & 2.124 & 4.932 & $\begin{array}{c}\text { Desviación } \\
\text { estándar }\end{array}$ \\
\hline Mínimo & 0 & 0 & 0 & 0 & 0 & 0 & 0 & 0 & Mínimo \\
\hline Máximo & 21 & 6 & 15 & 41 & 16 & 9 & 13 & 30 & Máximo \\
\hline
\end{tabular}


Tabla 19

Baremos de la Escala de Estilos Parentales para la sierra (Huanta, Cerro de Pasco, Cusco, Huánuco, Huaraz, Huancavelica y Huancayo)

\begin{tabular}{|c|c|c|c|c|c|c|c|c|c|}
\hline \multirow{2}{*}{$\begin{array}{l}\text { Percenti- } \\
\text { les }\end{array}$} & \multicolumn{4}{|c|}{$\begin{array}{l}\text { Puntajes directos: subescala de } \\
\text { los estilos parentales del padre }\end{array}$} & \multicolumn{4}{|c|}{$\begin{array}{l}\text { Puntajes directos: subescala de } \\
\text { los estilos parentales de la madre }\end{array}$} & \multirow{2}{*}{$\begin{array}{l}\text { Categoría } \\
\text { diagnóstica }\end{array}$} \\
\hline & $A b$ & So & In & ET & $A b$ & So & In & ET & \\
\hline 99 & $16-21$ & 6 & $14-15$ & $33-42$ & $13-18$ & $8-9$ & $12-15$ & $30-42$ & Alto \\
\hline 95 & $12-15$ & $4-5$ & $9-13$ & $23-32$ & 8-12 & 7 & $8-11$ & $23-29$ & Alto \\
\hline 90 & 11 & --- & 8 & $21-22$ & 7 & --- & 7 & $18-22$ & Alto \\
\hline 85 & $8-10$ & 3 & 7 & $18-20$ & 6 & 6 & 6 & $14-17$ & Medio \\
\hline 80 & 7 & --- & 6 & $14-17$ & 5 & 5 & 5 & $12-13$ & Medio \\
\hline 75 & 6 & --- & 5 & $12-13$ & 4 & --- & --- & 11 & Medio \\
\hline 70 & --- & --- & --- & 11 & --- & 4 & 4 & 10 & Medio \\
\hline 65 & 5 & 2 & 4 & $9-10$ & --- & --- & --- & 9 & Medio \\
\hline 60 & --- & --- & --- & --- & 3 & --- & 3 & --- & Medio \\
\hline 55 & 4 & --- & 3 & 8 & --- & --- & --- & --- & Medio \\
\hline 50 & 3 & 1 & 2 & 7 & 2 & 3 & 2 & 8 & Medio \\
\hline 45 & --- & --- & --- & 6 & --- & 2 & --- & 7 & Medio \\
\hline 40 & --- & --- & 1 & --- & 1 & --- & --- & 6 & Medio \\
\hline 35 & --- & 0 & --- & --- & --- & --- & 1 & --- & Medio \\
\hline 30 & 2 & --- & --- & 5 & --- & --- & --- & 5 & Medio \\
\hline 25 & --- & --- & --- & 4 & --- & 1 & --- & 4 & Medio \\
\hline 20 & --- & --- & --- & --- & 0 & --- & --- & --- & Medio \\
\hline 15 & 1 & --- & 0 & 3 & --- & --- & 0 & 3 & Medio \\
\hline 10 & --- & --- & --- & 2 & --- & --- & --- & 2 & Bajo \\
\hline 5 & 0 & --- & --- & $0-1$ & --- & 0 & --- & 1 & Bajo \\
\hline 1 & --- & --- & --- & --- & --- & --- & --- & 0 & Bajo \\
\hline $\mathrm{N}$ & 1216 & 1216 & 1216 & 1216 & 1216 & 1216 & 1216 & 1216 & $\mathrm{~N}$ \\
\hline Media & 4.56 & 1.31 & 3.24 & 9.11 & 2.82 & 2.98 & 2.87 & 8.66 & Media \\
\hline $\begin{array}{l}\text { Des- } \\
\text { viación } \\
\text { estándar }\end{array}$ & 3.710 & 1.379 & 3.111 & 7.284 & 2.927 & 2.008 & 2.770 & 6.355 & $\begin{array}{l}\text { Desviación } \\
\text { estándar }\end{array}$ \\
\hline Mínimo & 0 & 0 & 0 & 0 & 0 & 0 & 0 & 0 & Mínimo \\
\hline Máximo & 21 & 6 & 15 & 42 & 18 & 9 & 15 & 37 & Máximo \\
\hline
\end{tabular}


Tabla 20

Baremos de la Escala de Estilos Parentales para la selva (Bagua Grande, Moyobamba y Pucallpa)

\begin{tabular}{|c|c|c|c|c|c|c|c|c|c|}
\hline \multirow{2}{*}{$\begin{array}{l}\text { Percenti- } \\
\text { les }\end{array}$} & \multicolumn{4}{|c|}{$\begin{array}{l}\text { Puntajes directos: subescala de } \\
\text { los estilos parentales del padre }\end{array}$} & \multicolumn{4}{|c|}{$\begin{array}{l}\text { Puntajes directos: subescala de } \\
\text { los estilos parentales de la madre }\end{array}$} & \multirow{2}{*}{$\begin{array}{l}\text { Categoría } \\
\text { diagnóstica }\end{array}$} \\
\hline & $A b$ & So & In & ET & $A b$ & So & In & ET & \\
\hline 99 & $16-21$ & $5-6$ & $12-15$ & $30-42$ & $12-18$ & $8-9$ & $12-15$ & $30-42$ & Alto \\
\hline 95 & $9-15$ & --- & $9-11$ & $19-29$ & $7-11$ & $6-7$ & $8-11$ & $18-29$ & Alto \\
\hline 90 & $7-8$ & $3-4$ & $7-8$ & $15-18$ & $5-6$ & 5 & $6-7$ & $14-17$ & Alto \\
\hline 85 & 6 & --- & 6 & $12-14$ & --- & --- & 5 & $11-13$ & Medio \\
\hline 80 & --- & 2 & 5 & 11 & 4 & 4 & --- & 10 & Medio \\
\hline 75 & 5 & --- & --- & 10 & --- & --- & 4 & --- & Medio \\
\hline 70 & --- & --- & 4 & 9 & 3 & --- & --- & 9 & Medio \\
\hline 65 & --- & --- & --- & 8 & --- & --- & 3 & 8 & Medio \\
\hline 60 & 4 & --- & 3 & --- & --- & 3 & --- & --- & Medio \\
\hline 55 & --- & --- & --- & 7 & 2 & --- & --- & 7 & Medio \\
\hline 50 & 3 & 1 & 2 & 6 & 1 & 2 & 2 & 6 & Medio \\
\hline 45 & 2 & 0 & --- & 5 & --- & --- & 1 & --- & Medio \\
\hline 40 & --- & --- & 1 & --- & --- & --- & --- & 5 & Medio \\
\hline 35 & --- & --- & --- & 4 & --- & --- & --- & 4 & Medio \\
\hline 30 & --- & --- & --- & --- & --- & 1 & --- & --- & Medio \\
\hline 25 & 1 & --- & --- & 3 & 0 & --- & 0 & --- & Medio \\
\hline 20 & --- & --- & 0 & --- & --- & --- & --- & 3 & Medio \\
\hline 15 & --- & --- & --- & 2 & --- & --- & --- & 2 & Medio \\
\hline 10 & 0 & --- & --- & 1 & --- & --- & --- & --- & Bajo \\
\hline 5 & --- & --- & --- & 0 & --- & 0 & --- & 1 & Bajo \\
\hline 1 & --- & --- & --- & --- & --- & --- & --- & 0 & Bajo \\
\hline $\mathrm{N}$ & 492 & 492 & 492 & 492 & 492 & 492 & 492 & 492 & $\mathrm{~N}$ \\
\hline Media & 3.56 & .96 & 2.78 & 7.29 & 2.22 & 2.46 & 2.50 & 7.17 & Media \\
\hline $\begin{array}{l}\text { Des- } \\
\text { viación } \\
\text { estándar }\end{array}$ & 3.047 & 1.208 & 2.893 & 5.927 & 2.530 & 1.684 & 2.602 & 5.376 & $\begin{array}{l}\text { Desviación } \\
\text { estándar }\end{array}$ \\
\hline Mínimo & 0 & 0 & 0 & 0 & 0 & 0 & 0 & 0 & Mínimo \\
\hline Máximo & 18 & 6 & 15 & 34 & 16 & 9 & 14 & 36 & Máximo \\
\hline
\end{tabular}


